\title{
SAMPLING IN THERMOACOUSTIC TOMOGRAPHY
}

\author{
CHASE MATHISON
}

\begin{abstract}
We explore the effect of sampling rates when measuring data given by $M f$ for special operators $M$ arising in Thermoacoustic Tomography. We start with sampling requirements on $M f$ given $f$ satisfying certain conditions. After this we discuss the resolution limit on $f$ posed by the sampling rate of $M f$ without assuming any conditions on these sampling rates. Next we discuss aliasing artifacts when $M f$ is known to be under sampled in one or more of its variables. Finally, we discuss averaging of measurement data and resulting aliasing and artifacts, along with a scheme for anti-aliasing.
\end{abstract}

\section{INTRODUCTION}

This work builds on the theory laid out in [16] on sampling Fourier Integral Operators (FIOs). We discuss the specific application of Thermoacoustic Tomography, in which case the measurement operator $M$ is an FIO under suitable conditions. We discuss the theoretical resolution of $f$ given the sampling rate of $M f$ and then discuss aliasing and averaged data. Lastly we will show empirical evidence of our findings using numerical simulations.

Thermoacoustic Tomography is a medical imaging method in which a short pulse of electromagnetic radiation is used to excite cells in some object we wish to image, typically the organs of a patient. Upon absorbing the EM radiation, the cells in the patient in turn vibrate, creating ultrasonic waves that then propagate out of the patient and are measured by any number of methods. Using this measured data, we then try to reconstruct, in some sense, an image of the inside of the patient. This is a hybrid imaging method which uses high contrast, low resolution EM radiation to excite the cells; and low contrast, high resolution ultrasound waves as measurement [14, 11, 10, 9 , 21]. The hope is to be able to get an image with good contrast and resolution by combining these two types of waves.

More precisely, let $\Omega \subset \mathbb{R}^{n}$ be an open subset of Euclidean $n$-space such that $\bar{\Omega} \subset B_{R}(0)$ for some $R>0$ where $B_{R}(0)$ is the Euclidean ball of radius $R$. Suppose $f$ is a smooth function on $\mathbb{R}^{n}$ supported in $\Omega$. We view $f$ as the initial pressure distribution internal to some object to be imaged. Then, after exposing $\Omega$ to EM radiation, the ultrasonic waves created solve the acoustic wave equation:

$$
\begin{cases}\left(\partial_{t}^{2}-c^{2}(x) \Delta_{g_{0}}\right) u=0 & (t, x) \in[0, \infty) \times \mathbb{R}^{n}, \\ \left.u\right|_{t=0}=f(x) & x \in \mathbb{R}^{n}, \\ \left.\partial_{t} u\right|_{t=0}=0 & x \in \mathbb{R}^{n} .\end{cases}
$$

Here, $c(x)>0$ is the wave speed, which we take to be identically 1 outside of $K \subset \subset \Omega$. We assume that $c$ is a smooth function of $x$. In addition, $g_{0}$ is the Riemannian metric on the space $\bar{\Omega}$, assumed to be Euclidean on $\partial \Omega$. We define $g:=c^{-2} g_{0}$, which is the metric form which determines the geometry of this problem. Assume $u(t, x)$ is a solution to (1) for all $(t, x) \in[0, \infty) \times \mathbb{R}^{n}$. Further suppose that we have access to $u(t, y)$ for $(t, y) \in(0, T) \times \Gamma$ where $T>0$ and $\Gamma \subset \partial \Omega$ is a relatively

Date: December 11, 2019. 
open subset of $\partial \Omega$ (for this paper, we will take $\Gamma=\partial \Omega$ ). We define for $(t, y) \in(0, T) \times \Gamma$ the distribution $M f$ as the measurement operator:

$$
\begin{gathered}
M: C_{0}^{\infty}(\Omega) \rightarrow C_{(0)}^{\infty}((0, T) \times \Gamma), \\
M f(t, y)=u(t, y),(t, y) \in(0, T) \times \Gamma,
\end{gathered}
$$

where $C_{(0)}^{\infty}((0, T) \times \Gamma)$ is the space of smooth functions $\phi$ on $(0, T) \times \Gamma$ such that $\phi(t, y)=0$ near $t=0$. The methods used to collect data on $\Gamma$ are varied and include point detectors $12,17,8$, 7], integrating line detectors [2, 4], circular integrating detectors [6, 22], and 2D planar detectors [18, 5]. We note that at least when $f \in C_{0}^{\infty}(\Omega)$, by energy estimates, $M$ is well defined. We may actually even take $f$ to be a distribution in $\mathcal{D}^{\prime}(\Omega)$ such that $\|f\|_{H_{D}}=\int_{\Omega}|\nabla f|^{2} d x<\infty$, and by conservation of energy, $M$ extends to a well defined operator. The closure of $C_{0}^{\infty}(\Omega)$ under the previously stated norm is the space $H_{D}(\Omega) \subset H_{0}^{1}(\Omega)$, and we will assume $f \in H_{D}(\Omega)$ unless otherwise stated.

1.1. $M$ as an FIO. To obtain an oscillatory integral representation of $M$, we may use the geometric optics construction to solve for $u(t, x)$ in $(0, T) \times \mathbb{R}^{n}$ up to a smooth error (see [17, 20] for more details). This construction leads to the representation

$$
u(t, x)=\frac{1}{(2 \pi)^{n}} \sum_{\sigma= \pm} \int e^{i \phi_{\sigma}(t, x, \xi)} a_{\sigma}(t, x, \xi) \hat{f}(\xi) d \xi
$$

where $\phi_{\sigma}$ are solutions to the eikonal equation $\left(\partial_{t} \phi_{\sigma}\right)^{2}=c^{2}(x)\left|\nabla_{x} \phi_{\sigma}\right|_{g_{0}}^{2}$ with initial conditions $\phi_{\sigma}(0, x, \xi)=x \cdot \xi$. Note that solutions to the eikonal equation are local in nature, and so this representation of $u(t, x)$ is only valid until some time $T_{1}$. However, we may then solve (1) with "initial" conditions $\tilde{u}(0, x)=u\left(T_{1}, x\right)$ and $\left.\partial_{t} \tilde{u}(t, x)\right|_{t=0}=\left.\partial_{t} u(t, x)\right|_{t=T_{1}}$ using the same geometric optics construction. In this way, we can obtain an "approximate" solution to (1) for all $(t, x)$. Note by approximate, we mean up to a smooth error term. This error term could be quite large in the $L^{\infty}$ sense, but because it is a smooth term, it is negligible in the calculus of FIOs. It can be shown that $M=M_{+}+M_{-}$is a sum of elliptic FIOs of order 0 associated with locally diffeomorphic canonical relations that are each (locally) one-to-one mappings (see i.e. [16, 17]). We record the canonical relations $C_{+}$and $C_{-}$here for later use:

$$
C_{ \pm}:(x, \xi) \mapsto\left(s_{ \pm}(x, \xi), \gamma_{x, \xi}\left(s_{ \pm}(x, \xi)\right), \mp|\xi|_{g}, \dot{\gamma}_{x, \xi}^{\prime}\left(s_{ \pm}(x, \xi)\right)\right) .
$$

Here, we have $s_{ \pm}(x, \xi)$ is the exit time of the geodesic starting at $x$ in the direction $\pm g^{-1} \xi, \gamma_{x, \xi}(t)$ is the point on the geodesic issued from $(x, \xi)$ at time $t$ and $\dot{\gamma}_{x, \xi}^{\prime}(t)$ is the orthogonal (in the metric) projection of $\dot{\gamma}_{x, \xi}(t)$ onto $T \partial \Omega$ (the tangent bundle of the boundary of $\Omega$, so implicitly, we assume that $\partial \Omega$ is a at least a $C^{1}$ manifold). We assume that the metric induced by $g:=c^{-2}(x) g_{0}$ is non trapping, so that $\left|s_{ \pm}(x, \xi)\right|<\infty$ for all $(x, \xi) \in T^{*} \Omega$. Note that because each of the canonical relations $C_{+}$and $C_{-}$are one-to-one, the full canonical relation of the FIO $M$ given by $C=C_{+} \cup C_{-}$ is one-to-two, which makes intuitive sense as singularities split and travel along geodesics according to propagation of singularities theory.

\section{ACKnowledgments}

The author would like to thank Dr. Plamen Stefanov for suggesting this problem and for his guidance in the analysis of this problem. 


\section{Preliminary Definitions AND theorems}

2.1. Semiclassical analysis. The main definitions and theorems of semiclassical analysis and sampling that we use come from 23, 16. For a more complete background on semiclassical analysis, see [23. In sampling the measurement operator $M f$, we are interested in how the sampling rates affect our ability to resolve singularities with high frequency. To model this, we will rescale covectors $\xi$ by a factor of $1 / h$ where $h$ is a small parameter. We then examine families of functions (or distributions) $f_{h}$ that satisfy certain growth conditions as $h$ becomes small. Because of this, instead of considering the classical wave front set of a distribution, we consider the semiclassical wave front set, denoted $\mathrm{WF}_{h}(f)$. Note that $f$ is understood here to be a family of functions $f_{h}$ depending on the parameter $h$, but we will drop this subscript when it will not cause confusion. A key tool in analyzing the behavior of the measurement operator $M$ will be the semiclassical Fourier Transform, defined below.

Definition 2.1 (Semiclassical Fourier Transform). The semiclassical Fourier transform of an $h$ dependent family of distributions is defined as

$$
\mathcal{F}_{h} f_{h}(\xi)=\int e^{-i x \cdot \xi / h} f_{h}(x) d x
$$

If we denote the classical Fourier Transform by $\mathcal{F}$, then we have

$$
\mathcal{F}_{h} f_{h}(\xi)=\mathcal{F} f_{h}\left(\frac{\xi}{h}\right)
$$

Much like in classical analysis, we can use the semiclassical Fourier transform to define Sobolev norms on certain classes of functions or distributions.

Definition 2.2 ( $h$-Tempered family of distributions). The $h$-dependent family $f_{h}$ of distributions in $\mathcal{S}^{\prime}$ is said to be $h$-tempered if

$$
\left\|f_{h}\right\|_{H_{h}^{s}}^{2}:=(2 \pi h)^{-n} \int\langle\xi\rangle^{2 s}\left|\mathcal{F}_{h} f(\xi)\right|^{2} d \xi
$$

is such that $\left\|f_{h}\right\|_{H_{h}^{s}}=O\left(h^{-N}\right)$ for some $s$ and $N$. Here, we have $\langle\xi\rangle=\sqrt{1+|\xi|^{2}}$.

Another key tool we will use is the idea of the semiclassical wave front set of an $h$-dependent family of distributions.

Definition 2.3 (Semiclassical Wave Front Set). The semiclassical wave front set $\mathrm{WF}_{h}\left(f_{h}\right)$ of the $h$-tempered family $f_{h}$ is defined to be the complement of the set of $\left(x_{0}, \xi^{0}\right) \in \mathbb{R}^{2 n}$ such that there exists $\phi \in C_{0}^{\infty}\left(\mathbb{R}^{n}\right)$ with $\phi\left(x_{0}\right) \neq 0$ so that

$$
\mathcal{F}_{h}\left(\phi f_{h}\right)=O\left(h^{\infty}\right) \quad\left(\text { in } L^{\infty}\right)
$$

for $\xi$ in a neighborhood of $\xi^{0}$.

This set plays a similar role as the classical wave front set from microlocal analysis, however in general there is no sort of inclusion between these two sets. As an example [23], the coherent state

$$
f_{h}(x)=e^{-\left|x-x_{0}\right|^{2} /(2 h)} e^{i x \cdot \xi^{0} / h},
$$

has an empty wave front set in the classical sense, as it is a smooth function in both $x$ and $\xi$, however its semiclassical wave front set is $\mathrm{WF}_{h}\left(f_{h}\right)=\left\{\left(x_{0}, \xi^{0}\right)\right\}$. Note also that the zero section is allowed to be a part of the semiclassical wave front set, unlike in the classical case. Also, we do not require the semiclassical wave front set to be a conic set, which is another way that this set differs from the classical wave front set. 
We call elements of $\mathrm{WF}_{h}\left(f_{h}\right)$ singularities, even though a function with finite semiclassical wave front set is actually smooth.

Definition 2.4 ( $h-\Psi D O)$. We will use the standard quantization to define semiclassical pseudodifferential operators. Fix $m$ and $k \in \mathbb{R}$ and let $a(x, \xi) \in C^{\infty}\left(\mathbb{R}^{2 n}\right)$ satisfy the following: For every $\alpha$ and $\beta$ multi-indices and every compact set $K \subset \mathbb{R}^{n}$ there exists some $C_{\alpha, \beta, K}>0$ such that

$$
\left|D_{x}^{\alpha} D_{\xi}^{\beta} a(x, \xi)\right| \leq C_{\alpha, \beta, K} h^{k}\langle\xi\rangle^{m}
$$

for all $x \in K$ and $\xi \in \mathbb{R}^{n}$. We then say $a(x, \xi)$ is a semiclassical symbol of order $\leq m$. Then we define the semiclassical pseudodifferential operator $a(x, h D)$ by

$$
a(x, h D) f(x):=(2 \pi h)^{-n} \iint_{\mathbb{R}^{2 n}} e^{i(x-y) \cdot \xi / h} a(x, \xi) f(y) d y d \xi .
$$

Definition 2.5. The $h$-tempered family $f_{h}$ is said to be localized in phase space if there exists some $\psi \in C_{0}^{\infty}\left(\mathbb{R}^{2 n}\right)$ such that

$$
(\mathrm{Id}-\psi(x, h D)) f_{h}=O_{\mathcal{S}}\left(h^{\infty}\right)
$$

Note that because the functions we work with are semiclassically band limited (see definition 2.7), that all functions we work with can be assumed to be localized in phase space unless otherwise stated.

Definition 2.6 (Semiclassical Frequency Set). For each tempered $h$-dependent distribution $f_{h}$ localized in phase space, set

$$
\Sigma_{h}\left(f_{h}\right)=\left\{\xi \mid(x, \xi) \in \mathrm{WF}_{h}\left(f_{h}\right) \text { for some } x \in \mathbb{R}^{n}\right\} .
$$

This is simply the projection of $\mathrm{WF}_{h}\left(f_{h}\right)$ onto the second variable.

Definition 2.7 (Semiclassically Band Limited Functions). We say that $f_{h} \in C_{0}^{\infty}\left(\mathbb{R}^{n}\right)$ is semiclassically band limited (in $\mathcal{B})$ if

(1) $\operatorname{supp} f_{h}$ is contained in an $h$-independent set,

(2) $f_{h}$ is tempered,

(3) there exists a compact set $\mathcal{B} \subset \mathbb{R}^{n}$ such that for every open $U \supset \mathcal{B}$, we have for every $N$ there exists $C_{N}$ such that

$$
\left|\mathcal{F}_{h} f_{h}(\xi)\right| \leq C_{N} h^{N}\langle\xi\rangle^{-N} \text { for } \xi \notin U .
$$

Semiclassically band limited functions are those functions that can be reconstructed up to a smooth error from their samples, much like the band limited functions are those that can be perfectly reconstructed from their samples in the classical Nyquist Sampling theorem given a small enough sampling rate 13 .

2.2. Sampling. The main theorem used in [16] is the following:

Theorem 2.8. Assume that $\Omega \subset \mathbb{R}^{n}, \mathcal{B} \subset \mathbb{R}^{n}$ are open and bounded. Let $f_{h} \in C_{0}^{\infty}(\Omega)$ satisfy

$$
\left\|(I d-\psi(x, h D)) f_{h}\right\|_{H_{h}^{m}}=O\left(h^{\infty}\right)\left\|f_{h}\right\|, \quad \forall m \gg 0,
$$

for some $\psi \in C_{0}^{\infty}\left(\mathbb{R}^{2 n}\right)$ such that $\operatorname{supp}_{\xi} \psi \subset \mathcal{B}$. Let $\hat{\chi} \in L^{\infty}\left(\mathbb{R}^{n}\right)$ be such that $\operatorname{supp} \hat{\chi} \subset \mathcal{B}$ and $\hat{\chi}=1$ near $\operatorname{supp}_{\xi} \psi$.

Assume that $W$ is an invertible matrix so that the images of $\mathcal{B}$ under the translations $\xi \mapsto$ $\xi+2 \pi\left(W^{*}\right)^{-1} k, k \in \mathbb{Z}^{n}$, are mutually disjoint. Then for every $s \in(0,1]$,

$$
f_{h}(x)=|\operatorname{det} W| \sum_{k \in \mathbb{Z}^{n}} f_{h}(\operatorname{sh} W k) \chi\left(\frac{\pi}{s h}(x-s h W k)\right)+O_{H^{m}}\left(h^{\infty}\right)\left\|f_{h}\right\|_{L^{2}},
$$


for every $m \geq 0$, and

$$
\left\|f_{h}\right\|_{L^{2}}^{2}=|\operatorname{det} W|(s h)^{n} \sum_{k \in \mathbb{Z}^{n}}\left|f_{h}(s h W k)\right|^{2}+O\left(h^{\infty}\right)\|f\|_{L^{2}}^{2} .
$$

The proof of this theorem essentially follows from the classical Nyquist sampling theorem and can be found in [16, 15]. For all applications in this paper, we take the matrix $W$ above to be the identity matrix.

We make heavy use of the following theorem which relates how classical FIOs effect semiclassical wavefront sets from [16], where the reader can find the proof.

Theorem 2.9. Let $A$ be an FIO in the class $I^{m}\left(\mathbb{R}^{n_{2}}, \mathbb{R}^{n_{1}}, \Lambda\right)$ where $\Lambda \subset T^{*}\left(\mathbb{R}^{n_{1}} \times \mathbb{R}^{n_{2}}\right) \backslash 0$ is a Lagrangian manifold and $m \in \mathbb{R}$. Then for every $f_{h}$ localized in phase space,

$$
\mathrm{WF}_{h}(A f) \backslash 0 \subset C \circ \mathrm{WF}_{h}(f) \backslash 0,
$$

where $C=\Lambda^{\prime}$ is the canonical relation of $A$.

This theorem shows how classical FIOs affect the semiclassical wavefront set away from the zero section. In particular, the semiclassical wavefront set of $A f$ away from the zero section transforms in the same way the classical wavefront set does: it is transformed by the canonical relation associated with $A$. The main assertion in [16] is that the sampling requirements of $M f$ given $\mathrm{WF}(f)$ are determined by $C$, the canonical relation associated with $M f$.

\section{Resolution Limit of $f$ Given SAMPLing RAte of $M f$}

Suppose we wish to sample the $M f$ at some fixed sampling rates $s_{t}$ and $s_{y^{j}}$. Here we don't assume that we know any information about $\Sigma_{h}(f)$, we only wish to see how fixing a sampling rate on $M f$ affects our ability to resolve singularities of $f$. Avoiding aliasing of $M f$ is equivalent to (by Theorem 2.8

$$
(\tau, \eta) \in \Sigma_{h}(M f) \Longrightarrow|\tau| \leq \frac{\pi}{s_{t}}, \quad\left|\eta_{j}\right| \leq \frac{\pi}{s_{y^{j}}}
$$

where $\tau$ is the dual variable to $t$, and $\eta$ is the dual variable to $y$, with $\eta_{j}$ the $j$ th component of $\eta$. Note that the norms $|\tau|$ and $|\eta|$ are taken in the corresponding metric. In particular, although the norm $g_{0}$ on $\bar{\Omega}$ is assumed to be Euclidean, the induced norm on the tangent space to the boundary, which we'll call $g_{0, \partial \Omega}$, is not necessarily Euclidean. We may use the canonical relation (2) $C$ associated with $M$ to write the inequalities above as

$$
|\xi|_{g}=\sqrt{c^{2} g_{0}^{i j} \xi_{i} \xi_{j}} \leq \frac{\pi}{s_{t}}, \quad\left|\dot{\gamma}_{x, \xi}^{\prime}\left(s_{ \pm}(x, \xi)\right)_{j}\right|_{g_{0, \partial \Omega}} \leq \frac{\pi}{s_{y^{j}}} .
$$

From this we can see that we have that avoiding aliasing is equivalent to

$$
c(x)|\xi|_{g_{0}} \leq \frac{\pi}{s_{t}}, \quad\left|\dot{\gamma}_{x, \xi}^{\prime}\left(s_{ \pm}(x, \xi)\right)_{j}\right|_{g_{0, \partial \Omega}} \leq \frac{\pi}{s_{y^{j}}}
$$

For most of the paper, we will assume that $g_{0}$ is Euclidean, although more general results hold.

3.1. The effect of $s_{t}$ on resolution. Consider the first inequality in (7) and assume that $s_{y^{j}}$ is taken small enough so as to not effect resolution of singularities of $f$. The first inequality indicates that the sampling rate $s_{t}$ imposes a limit on the resolution of $f$ such that for fixed $x$, there will be higher resolution of singularities of $f$ at points $(x, \xi)$ where the wave speed $c(x)$ is slower, and likewise the resolution will be worse at those points $(x, \xi)$ where the wave speed is faster. In 
particular, given the relative sampling rate $s_{t}$, we cannot resolve singularities at $x$ with frequency greater than

$$
|\xi|=\frac{\pi}{c(x) s_{t}}
$$

This is a local result. A global estimate for the maximum frequency of a singularity that is guaranteed to be resolved anywhere given the sampling rate $s_{t}$ is given by

$$
|\xi|=\frac{\pi}{c_{\max } s_{t}} .
$$

This is illustrated in Figures 1 and 2 below.

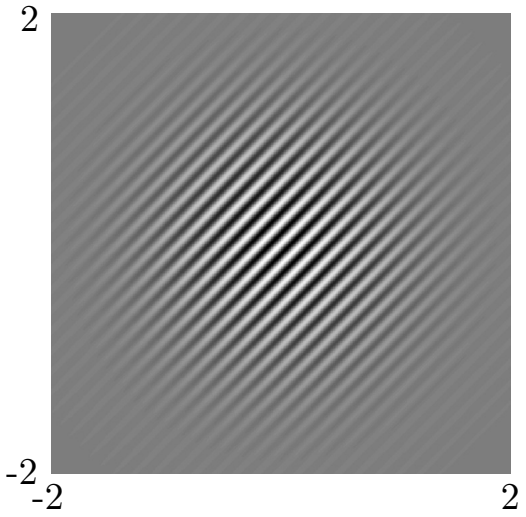

Original Image

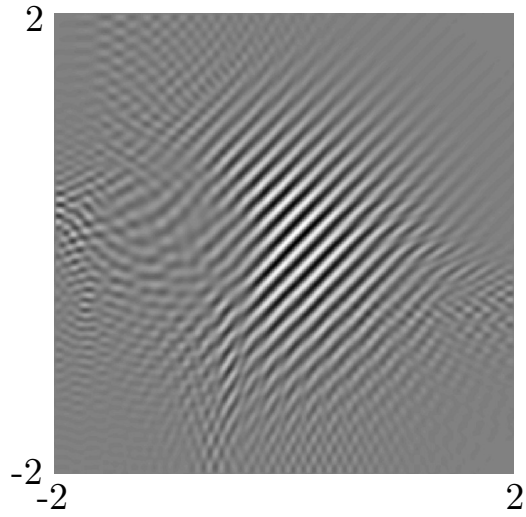

Time Reversal Reconstruction

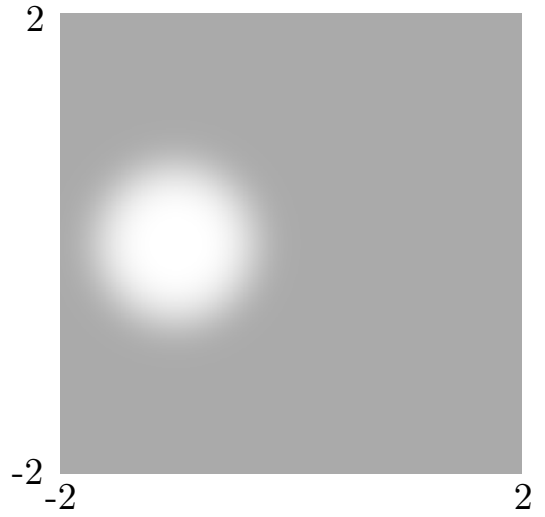

Wave Speed with Fast Spot

Figure 1. Resolution of $f$ given a fixed sampling rate $s_{t}$ of $M f(t, y)$. The wave speed here $c(x, y)=1+0.5 \exp \left(-\left((x+1)^{2}+y^{2}\right)^{2} / 0.25\right)$ has a fast spot centered at $x=-1$. We can see that this is precisely where the reconstruction of $f$ has poor resolution when under sampled in the $t$ variable, as explained above.

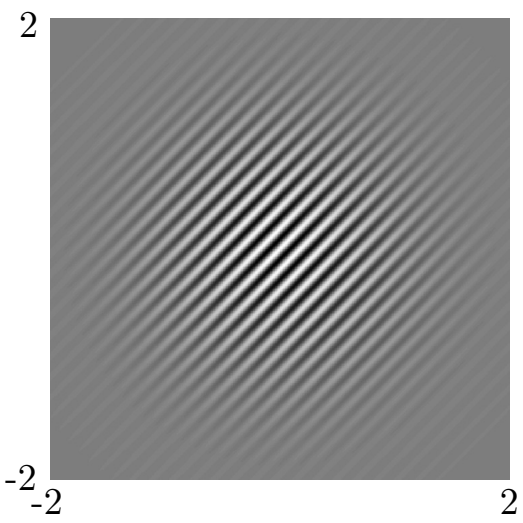

Original Image

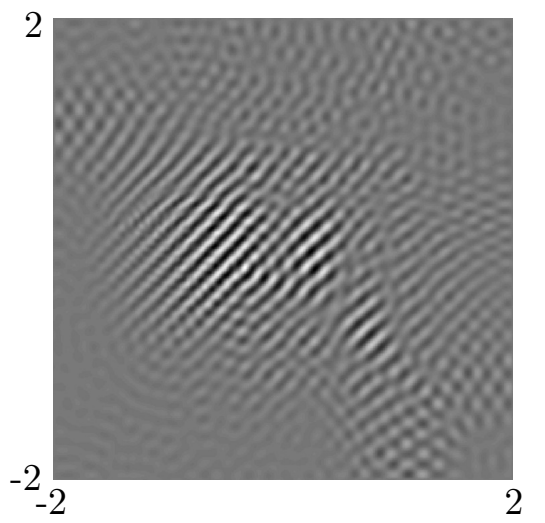

Time Reversal Reconstruction

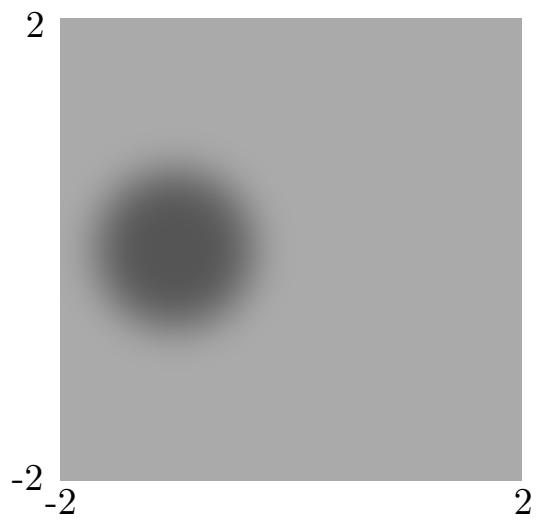

Wave Speed with Slow Spot

Figure 2. Resolution of $f$ given a fixed sampling rate $s_{t}$ of $M f(t, y)$. The wave speed here $c(x, y)=1-0.5 \exp \left(-\left((x+1)^{2}+y^{2}\right)^{2} / 0.25\right)$ has a slow spot centered at $x=-1$. We can see that this is precisely where the reconstruction of $f$ has the best resolution when under sampled in the $t$ variable, as explained above. 
3.2. The effect of $s_{y^{j}}$ on resolution. Assume now that $s_{t}$ is chosen small enough so as to not effect resolution of singularities of $f$. The second inequality in (7)

$$
\left|\dot{\gamma}_{x, \xi}^{\prime}\left(s_{ \pm}(x, \xi)\right)_{j}\right|_{g_{0}, \partial \Omega} \leq \frac{\pi}{s_{y^{j}}}
$$

tells us that the sampling rate $s_{y^{j}}$ imposes a limit on the resolution of $f$ such that singularities $(x, \xi)$ that intersect the boundary $\partial \Omega$ nearly perpendicularly will have higher resolution than those that hit the boundary nearly tangentially (at a large angle to the normal vector to $\partial \Omega$ at the point of intersection). Also, because $\left|\dot{\gamma}_{x, \xi}(t)\right|_{g}$ is constant along the geodesic $\gamma_{x, \xi}$, we know in particular that $\left|\dot{\gamma}_{x, \xi}^{\prime}\left(s_{ \pm}(x, \xi)\right)_{j}\right|_{g_{0, \partial \Omega}} \leq\left|\dot{\gamma}_{x, \xi}^{\prime}\left(s_{ \pm}(x, \xi)\right)\right|_{g_{0, \partial \Omega}}=|\xi|_{g} \cos (\theta)$ where $\theta$ is the angle (in the metric) between $\dot{\gamma}_{x, \xi}\left(s_{ \pm}(x, \xi)\right)$ and $\dot{\gamma}_{x, \xi}^{\prime}\left(s_{ \pm}(x, \xi)\right)$. This tells us that to avoid aliasing, we must have

$$
|\xi|_{g} \cos (\theta) \leq \frac{\pi}{s_{y^{j}}}
$$

We recall that $|\xi|_{g}^{2}=c^{2}(x) g_{0}^{i j} \xi_{i} \xi_{j}$, and in the case that $g_{0}$ is Euclidean, we get

$$
c(x)|\xi| \cos (\theta) \leq \frac{\pi}{s_{y^{j}}} .
$$

For a fixed relative sampling rate $s_{y^{j}}$, we cannot resolve singularities $(x, \xi)$ of $f$ of frequency greater than

$$
|\xi|=\frac{\pi}{s_{y^{j}} c(x) \cos (\theta)} .
$$

Note in particular that if $\theta=\frac{\pi}{2}$ (i.e. the geodesic $\gamma_{x, \xi}$ hits the boundary $\partial \Omega$ perpendicularly), then $c(x)|\xi| \cos (\theta)=0<\pi / s_{y^{j}}$, and we will always be able to resolve the singularity at $(x, \xi)$. Also note that this is a local result, and as is the case for $s_{t}$ "slow spots" in the speed $c(x)$ give better resolution of singularities in general. Because $c(x) \leq c_{\max }$, we also get the following estimate for the maximum frequency of a resolvable singularity, regardless of location:

$$
|\xi|=\frac{\pi}{c_{\max } s_{y^{j}} \cos (\theta)} .
$$

Finally, because $0<\theta \leq \pi / 2$, we know $0 \leq \cos (\theta)<1$, and we have the following (worst case) global estimate for the maximum frequency of a singularity of $f$ that can be resolved:

$$
|\xi|=\frac{\pi}{c_{\max } s_{y^{j}}}
$$

We note that if one wants to be able to resolve singularities of $f$ with frequency $K$, then by considering (8) and (9), the sampling rates $s_{t}$ and $s_{y^{j}}$ of $M f$ should be taken to be at least

$$
s_{t}=s_{y^{j}} \leq \frac{\pi}{K c_{\max }}
$$

where $c_{\max }$ is defined as before. In particular, we recover the result from 16 that for a semiclassically band limited $f_{h}$ with essential maximum frequency $B$ in the Euclidean case that we need to take sampling rates of $M f$ satisfying

$$
s_{t} \leq \frac{\pi}{B c_{\max }}, \quad s_{y^{j}} \leq \frac{\pi}{B c_{\max }},
$$

to avoid aliasing. These effects are shown in Figure 3 . 
3.3. CFL condition. We can relate this analysis to numerical solvers of the wave equation. When solving the wave equation numerically, a typical approach is to discretize the space and time domain, and use a finite difference scheme. Suppose we wish to simulate an experiment using a rectangular grid in the space coordinates and we collect data on the boundary of a square. Further, we assume that $g_{0}$ is Euclidean, and because the boundary is a rectangle, also the metric induced on the boundary is Euclidean. Suppose we have fixed each $s_{x^{j}}=\Delta x^{j} / h \leq \pi /\left(B c_{\max }\right)$ with a common value $s_{x}=\Delta x / h$, where $B$ is the essential band limit on $f$, i.e. $\Sigma_{h}(f) \subset[-B, B]^{n}$. Note that by our choice of $s_{x}$, there will not be aliasing of $M f$, provided $s_{t}$ is chosen well, as on the boundary in this rectangular grid, we have $s_{y}=s_{x}$, where all of the $s_{y^{j}}$ as above have a common fixed step size $s_{y}$. In order to choose $s_{t}$, we recall that the frequency set $\Sigma_{h}(M f)$ is contained in the set $\{(\tau, \eta)|| \eta|\leq| \tau \mid\}$. Because $f$ has a semiclassical band limit of $B$, we know that $\pi_{2}\left(\Sigma_{h}(M f)\right) \subset\left\{|\eta| \leq \sqrt{n} B c_{\max }\right\}$, where $\pi_{2}$ is the projection onto the second factor. We know this because each $\left|\eta_{j}\right| \leq B c_{\max }$. Also, by the analysis above, we know that $|\tau|=|\xi|_{g}$, but $|\xi|_{g} \leq \max |\xi| c_{\max }$. We also know that $\max |\xi|<\sqrt{n} B$, so that the largest possible size of $|\tau|$ given the band limit on $f$, is $\sqrt{n} B c_{\max }$. It is then clear that we need $\left.s_{t} \leq \pi /\left(\sqrt{n} B c_{\max }\right)\right)$ to avoid aliasing. This tells us that we should take $\Delta t \leq \pi h /\left(\sqrt{n} B c_{\max }\right)=\Delta x / \sqrt{n}$. Now, the CFL condition for the leapfrog finite difference scheme $([3,1,19])$ tells us that given a step size $\Delta x$ and wave speed $c(x)$, that we should take the time step $\Delta t \leq \Delta x /\left(\sqrt{n} c_{\max }\right)$ to ensure stability of the finite difference scheme. But $\Delta x /\left(\sqrt{n} c_{\max }\right) \leq \Delta x / \sqrt{n}$, because $c_{\max } \geq 1$. This means, that if we've chosen $\Delta x \leq \pi h /\left(B c_{\max }\right)$, and we choose $\Delta t$ satisfying the CFL condition for the leapfrog finite difference scheme, then there will be no aliasing in the measured data $M f$ at the boundary. Also, if $c_{\max }=1$, then the CFL condition is identical to the conditions on $\Delta x$ and $\Delta t$ required to avoid aliasing of the measured data $M f$.

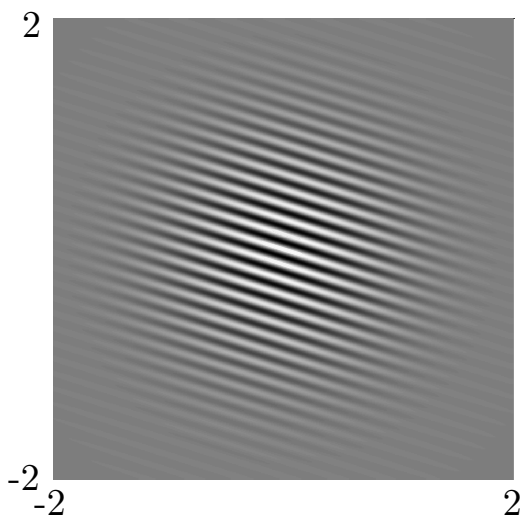

Original Image

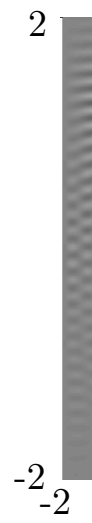

Time Reversal Reconstruction

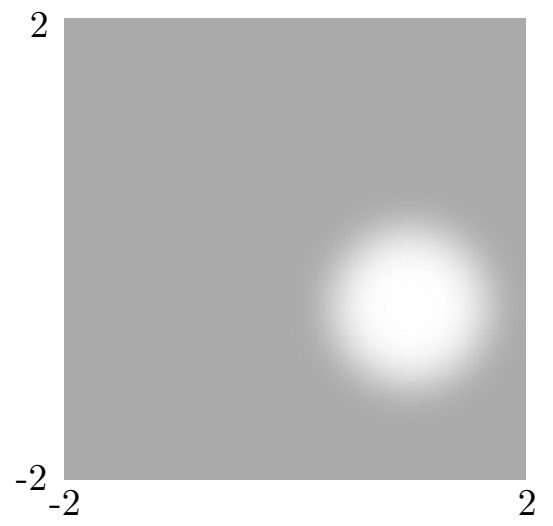

Wave Speed with Fast Spot

Figure 3. Resolution of $f$ given a fixed sampling rate $s_{y^{j}}$ of the space variables on the boundary $\partial \Omega$. We can see that the blurring effect is roughly uniform for points near the fast spot in the wave speed $\left.c(x, y)=1+0.5 \exp \left(-\left((x-1)^{2}+(y+0.5)^{2}\right)^{2}\right) / 0.25\right)$, but that there are singularities in the region where $c \approx 1$ far from the fast spot that are also highly affected. These singularities hit the boundary with a larger angle to the outward pointing normal vector, and so we expect lower resolution there.

\section{Aliasing and artifacts}

Now suppose that we know that $f_{h}$ is a semiclassically band limited function with essential band limit $B$. In [16], it is shown that in order to avoid aliasing of $M f_{h}$, for a semiclassically band limited 
$f_{h}$, we must have relative sample rates of $s_{t} \leq \frac{\pi N}{B}$ and $s_{y^{j}} \leq \frac{\pi N N^{\prime}}{B}$ where $B$ is half the side length of a box bounding $\Sigma_{h}(f), N$ is the sharp lower bound of the metric form $g=c^{-2} g_{0}$ on the unit sphere for all $x$, and $\left(N^{\prime}\right)^{2}$ is the sharp upper bound on the induced metric on the Euclidean sphere in a fixed chart for $y$. In the numerical examples that follow, $\partial \Omega$ is piecewise flat and parameterized in a Euclidean way, so that $N^{\prime}=1$ away from corners. Note that if $g_{0}$ is Euclidean, then setting $c_{\max }=\max c(x)$, we have $N=1 / c_{\max }$, and $N^{\prime}=1$ so that the relative sampling rates needed to avoid aliasing are

$$
s_{t} \leq \frac{\pi}{B c_{\max }}, \quad s_{y^{j}} \leq \frac{\pi}{B c_{\max }} .
$$

4.1. Under sampling in $t . s_{t}>\frac{\pi}{B c_{\max }}$. Then, by [16] there will be aliasing of $M f$. The error in the reconstruction can be modeled by the frequency shift operator

$$
S_{k}: \tau \rightarrow \tau+\frac{2 \pi k}{s_{t}} \text {. }
$$

This operator is valid as long as $\tau+2 \pi k / s_{t} \in\left[-\pi / s_{t}, \pi / s_{t}\right]$ (see Figure 4 (right)). If we have not

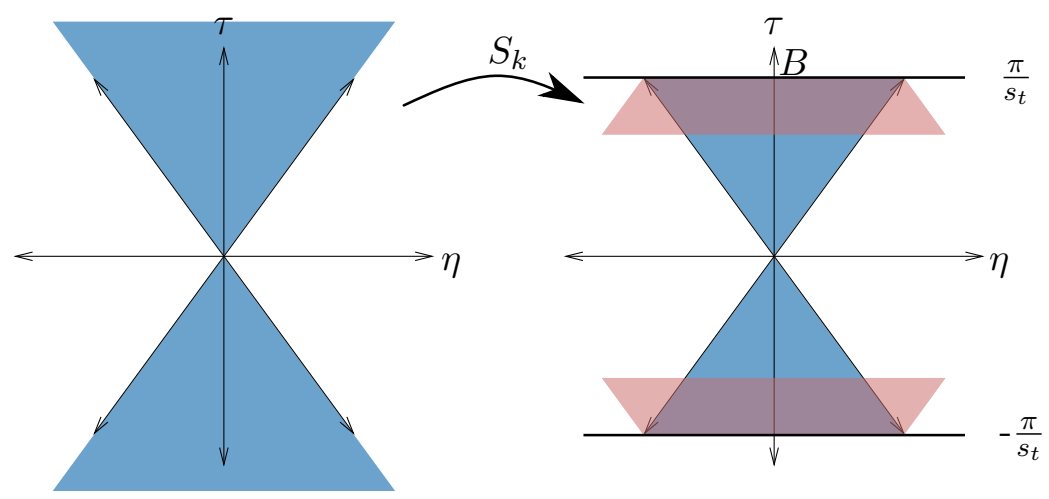

Figure 4. The characteristic cone in which $\Sigma_{h}(M f)$ must lie. The cone on the left shows the possible range of the covector $(\eta, \tau)$ which is determined by the canonical relation associated with $M$. The image on the right shows the possible range of covectors $(\eta, \tau)$ after under sampling (in $t$ ). Note that the red regions have been shifted up and down from the original frequency set by translation due to under sampling.

under sampled $M f$ too critically in the $t$ variable, we would expect to only see this added error for $k=-1,1$, with more terms added as the under sampling becomes worse. As explained in [16], by Egorov's Theorem, we expect to see artifacts in a reconstruction of $f$ that can be calculated by the canonical relation

$$
C_{ \pm}^{-1} \circ S_{k} \circ C_{ \pm}:(x, \xi) \rightarrow(\tilde{x}, \tilde{\xi})
$$

where $\tilde{x}$ and $\tilde{\xi}$ can be calculated by finding the operator on the left. We do that now for $C_{+}$:

$$
\begin{aligned}
C_{+}^{-1} \circ S_{k} \circ C_{+}(x, \xi) & =C_{+}^{-1} \circ S_{k}\left(s_{+}(x, \xi), \gamma_{x, \xi}\left(s_{+}(x, \xi)\right),-|\xi|_{g}, \dot{\gamma}_{x, \xi}^{\prime}\left(s_{+}(x, \xi)\right)\right) \\
& =C_{+}^{-1}\left(s_{+}(x, \xi), \gamma_{x, \xi}\left(s_{+}(x, \xi)\right),-|\xi|_{g}+\frac{2 \pi k}{s_{t}}, \dot{\gamma}_{x, \xi}^{\prime}\left(s_{+}(x, \xi)\right)\right) \\
& =\left(\gamma_{y,-\zeta}\left(s_{+}(x, \xi)\right),-\dot{\gamma}_{y,-\zeta}\left(s_{+}(x, \xi)\right)\right)
\end{aligned}
$$


where $y=\gamma_{x, \xi}\left(s_{+}(x, \xi)\right)$ is the point of intersection of the geodesic issued from $(x, \xi)$ with $\partial \Omega$, and $\zeta=\dot{\gamma}_{x, \xi}^{\prime}\left(s_{+}(x, \xi)\right)+\beta_{k} \eta^{\perp}$ where $\beta_{k}=\sqrt{\left(|\xi|_{g}-2 \pi k / s_{t}\right)^{2}-\left|\dot{\gamma}_{x, \xi}^{\prime}\left(s_{+}(x, \xi)\right)\right|^{2}}$ and $\eta^{\perp}=\dot{\gamma}_{x, \xi}\left(s_{+}(x, \xi)\right)-$ $\dot{\gamma}_{x, \xi}^{\prime}\left(s_{+}(x, \xi)\right)$. Aliasing artifacts are found using this mapping in Figures 5 and 6 below. The mapping $C_{-}^{-1} \circ S_{k} \circ C_{-}$is calculated in almost an identical fashion, however we have a change in sign in the $\tau$ variable.

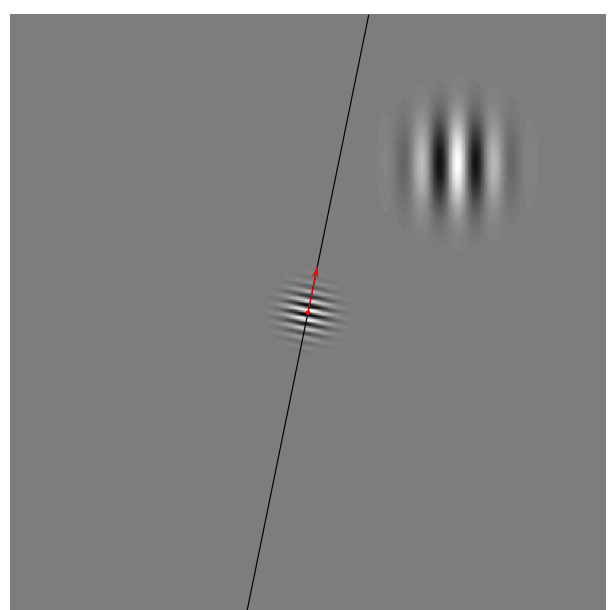

Original Image

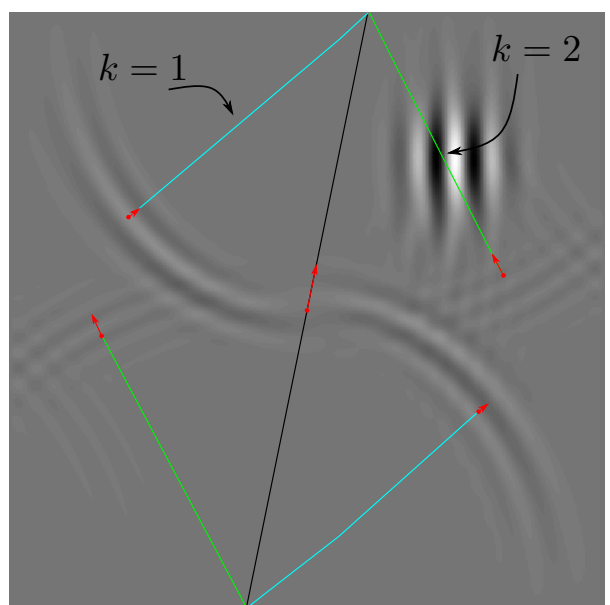

Reconstructed Image

Figure 5. Tracing the aliasing artifacts by using geodesics. We have used the constant wave speed $c \equiv 1$ for this example. Here we have under sampled in $t$ and show the image of the singularity $(x, \xi)$ under the canonical relations given by $C_{ \pm}^{-1} \circ S_{i} \circ C_{ \pm}$for $i=1,2$. Note that the low frequency singularity does not cause artifact, but the high frequency singularity vanishes in the reconstruction and causes aliasing artifacts.

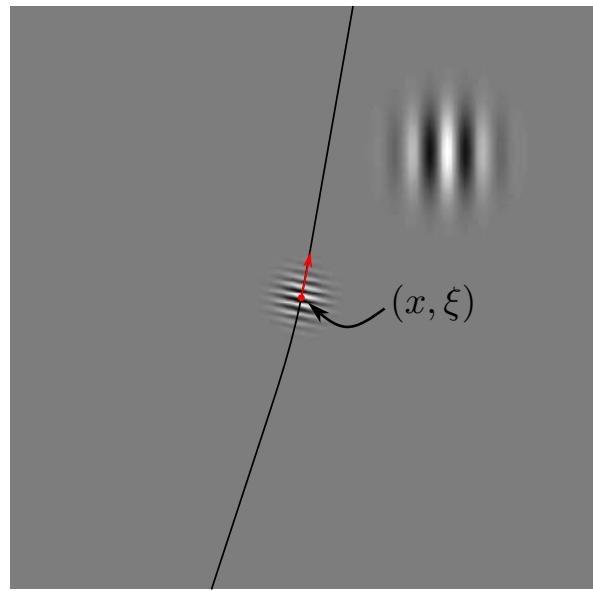

Original Image

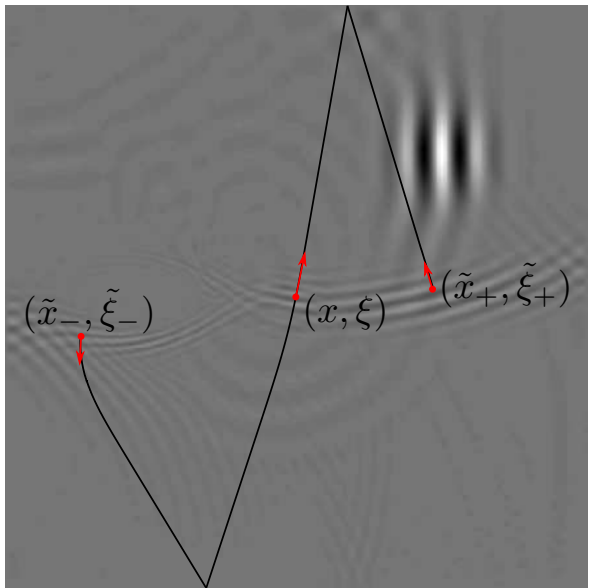

Reconstructed Image

Figure 6. Artifacts in a reconstructed image with $M f$ under sampled in time variable and a variable wave speed. We trace the geodesics to find the image of $(x, \xi)$ under the map $C_{ \pm} \circ S_{k} \circ C_{ \pm}$as explained above. 
We include a more complicated image reconstruction in Figure 16 along with the collected data in Figure 17. We also show how a smooth approximation of an line segment is affected by these artifacts in the image given in Figure 9. For this image and reconstruction, we have included the collected data and Fourier transform images in Figure 10.

4.2. Under sampling in $y$. Now suppose that we have under sampled the $y$ variable, i.e. we have chosen $s_{y^{j}}>\frac{\pi}{B}$ for some $j=1, \ldots, n$. Then again, we will have aliasing and the error in the reconstruction will involve the frequency shift operator, but now $S_{k}$ will act on $\eta_{j}$ as

$$
S_{k}: \eta_{j} \mapsto \eta_{j}+\frac{2 \pi k}{s_{y^{j}}} .
$$

This operator is valid as long as $\eta_{j}+\frac{2 \pi k}{s_{y^{j}}} \in\left[-\pi / s_{y^{j}}, \pi / s_{y^{j}}\right]$. The canonical relation of the $h$-FIO that operates on $M f$ as a reconstruction of $f$ will then be given by (again, we only consider $C_{+}$ here)

$$
C_{+}^{-1} \circ S_{k} \circ C_{+}(x, \xi)=C_{+}^{-1}\left(s_{+}(x, \xi), \gamma_{x, \xi}\left(s_{+}(x, \xi)\right),-|\xi|_{g}, \dot{\gamma}_{x, \xi}^{\prime}\left(s_{+}(x, \xi)\right)+\frac{2 \pi k}{s_{y^{j}}} \mathbf{e}_{j}\right)
$$

where $\mathbf{e}_{j}$ is the unit vector in the $y^{j}$ direction. Note that, in particular, this implies that the artifacts will have the same frequency as that of the original image, but perhaps with a space shift. Also, because this operator is valid as long as $\eta_{j}+2 \pi k / s_{y^{j}} \in\left[-\pi / s_{y^{j}}, \pi / s_{y^{j}}\right]$, if the geodesic emanating from $(x, \xi)$ hits the boundary $\partial \Omega$ perpendicularly, then the point $(x, \xi)$ will be unaffected by this shift in the reconstruction, i.e. there will be no artifacts that come from $(x, \xi)$. This is true because if the geodesic emanating from $(x, \xi)$ hits $\partial \Omega$ perpendicularly, then $\eta_{j}=0$ and $2 \pi k / s_{y^{j}} \notin\left[-\pi / s_{y^{j}}, \pi / s_{y^{j}}\right]$ for any $k \neq 0$. Finding these artifacts in practice follows in much the same way as finding where artifacts occur for under sampling in the time variable. We illustrate this for the constant speed, Euclidean case in Figure 7 and see Figure 8 for the variable speed case.

We again include a more complicated image reconstruction in Figure 18 along with the collected data in Figure 19. We also show how a smooth approximation of an line segment is affected by these artifacts in the image given in Figure 11. For this image and reconstruction, we have included the collected data and Fourier transform images in Figure 12.

\section{Averaged DATA}

Suppose that the collected data $M f(t, y)$ has been averaged in the $t$ or $y$ variables for some reason (in practice this can be done to try to avoid aliasing, or in an attempt to reduce the noise in data). This can be modeled in a few ways, including taking a convolution $\phi_{h} * M f$ with a smooth function $\phi_{h}=h^{n} \phi(\cdot / h)$ that decreases away from the origin to 0 . To model localized averaging however, we will consider data of the form $Q_{h} M f(t, y)$, where $Q_{h}$ is an $h$ - $\Psi$ DO with a principal symbol of the form $q_{0}(t, y, \tau, \eta)=\psi\left(a|\tau|^{2}+b|\eta|^{2}\right)$ where $\psi \in C_{0}^{\infty}(\mathbb{R})$ is decreasing. The effect of $Q_{h}$ is to limit $\mathrm{WF}_{h}(M f)$, which will in principle remove the high frequency singularities of $M f$ which will have a smoothing effect. From [16], we know that because $M$ is a FIO associated with the canonical map $C=C_{+} \cup C_{-}$, that the composition $Q_{h} M f$ can be written

$$
Q_{h} M f=M P_{h} f+O\left(h^{\infty}\right) f
$$




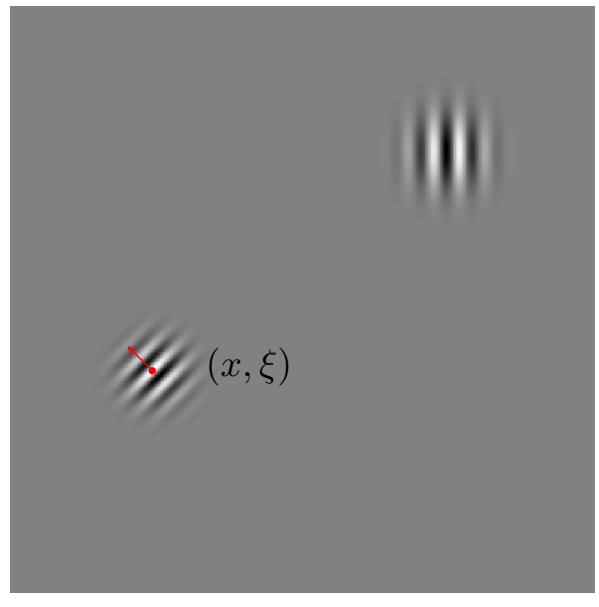

Original Image

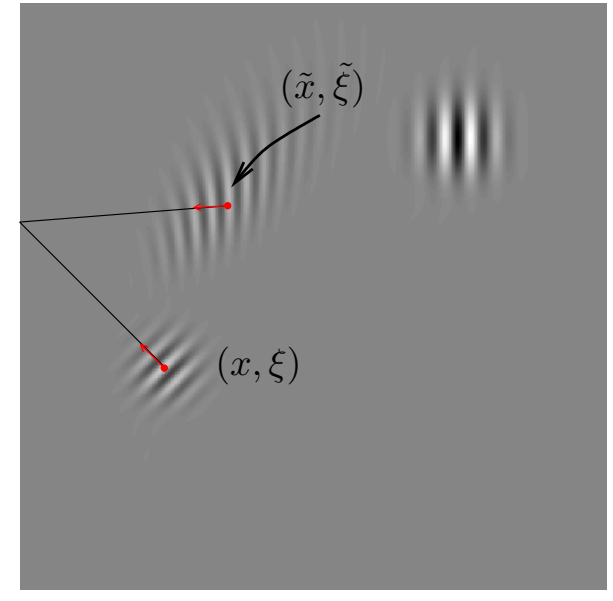

Reconstructed Image

Figure 7. Artifacts in a reconstructed image with $M f$ under sampled in space variables. Here we take $c \equiv 1$. Specifically, $M f$ here was under sampled on the left and right edges of the square. Note that there is no artifact in the reconstructed image coming from the pattern in the upper right corner of the square, because singularities from this pattern hit the boundary of the square perpendicularly. Note also that the original singularity still remains with half its amplitude because we did not under sample along the bottom edge of the square.

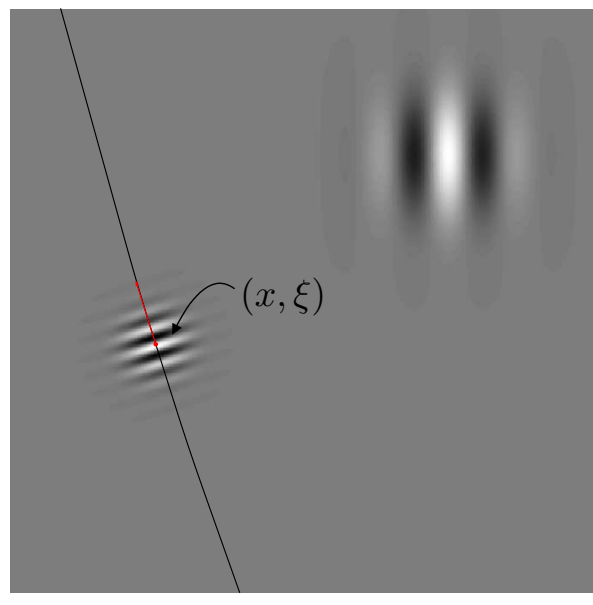

Original Image

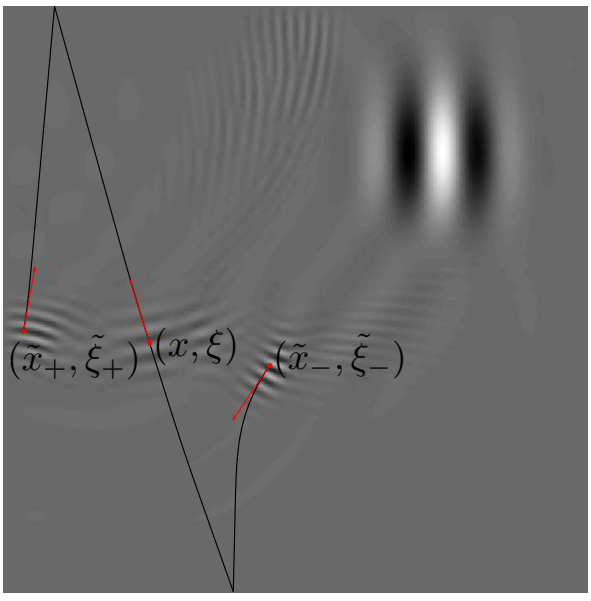

Reconstructed Image

Figure 8. Artifacts in a reconstructed image with $M f$ under sampled in space variables and a variable wave speed. Specifically, $M f$ here was under sampled on the top and bottom edges of the square. The artifacts in the reconstruction have the same frequency as the original, but with a space shift due to under sampling.

where $P_{h}$ is a $h-\Psi D O$ with principal symbol $p_{0}=q_{0} \circ C$ where $q_{0}$ is the principal symbol of $Q_{h}$. So, for $Q_{h}, q_{0}$, we may calculate

$$
\begin{aligned}
p_{0}(x, \xi) & =\frac{1}{2}\left(q_{0} \circ C_{+}(x, \xi)+q_{0} \circ C_{-}(x, \xi)\right) \\
& =\frac{1}{2}\left(\psi\left(a|\xi|_{g}^{2}+b\left|\dot{\gamma}_{x, \xi}^{\prime}\left(s_{+}(x, \xi)\right)\right|_{g_{0, \partial \Omega}}^{2}\right)+\psi\left(a|\xi|_{g}^{2}+b\left|\dot{\gamma}_{x, \xi}^{\prime}\left(s_{-}(x, \xi)\right)\right|_{g_{0, \partial \Omega}}^{2}\right)\right) .
\end{aligned}
$$




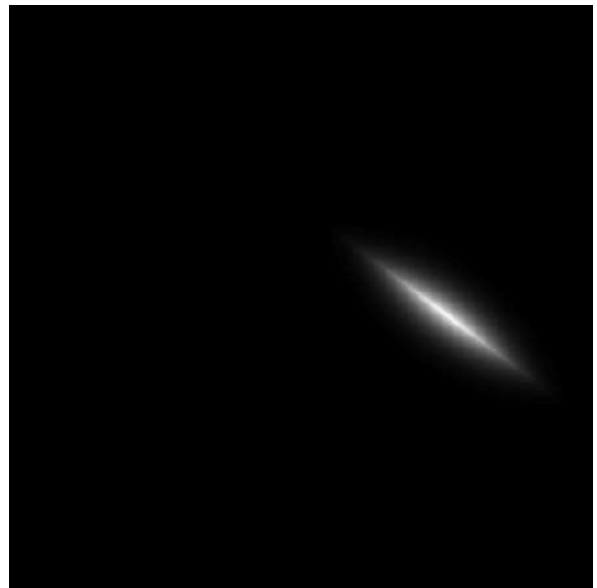

Original Image

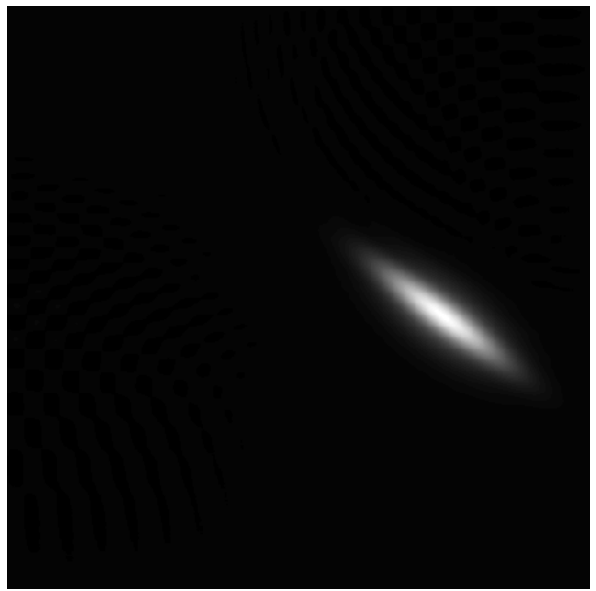

Reconstructed Image

Figure 9. Original and reconstructed image of a smooth approximation of an line segment. Here we have under sampled in $t$. The under sampling has resulted in blurring of this "line segment". This is due to the fact that under sampling in $t$ shifts high frequency data in $\mathcal{F}(M f)$.

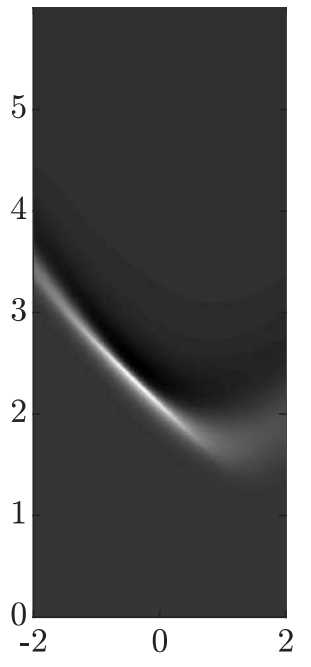

Collected Data

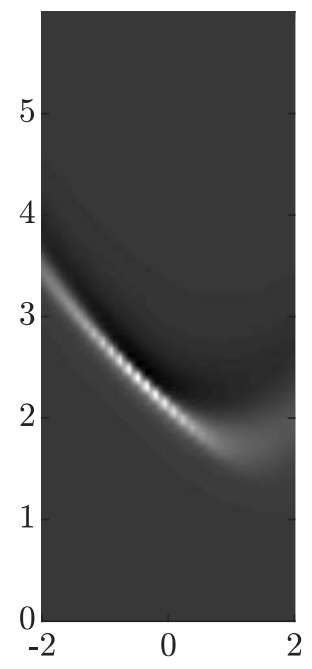

Downsampled Data
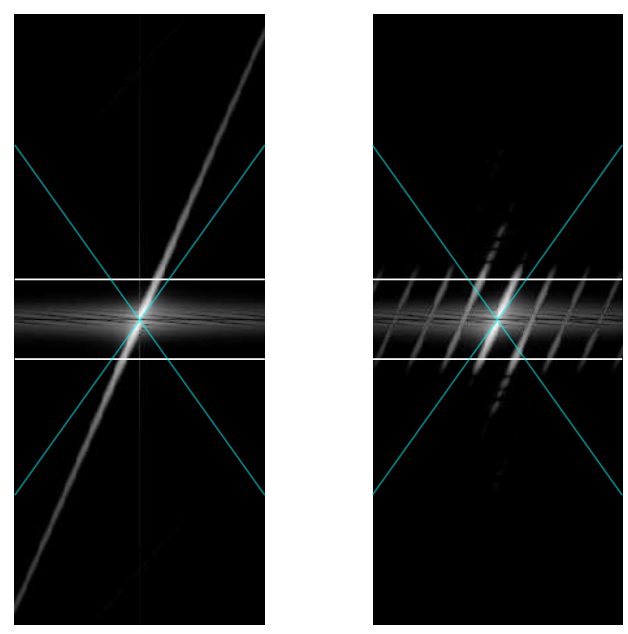

Fourier Transform Fourier Transform of of Collected Data Undersampled Data

Figure 10. Collected data and Fourier transform along with under sampled data in $t$ for example given in Figure 9. Data was collected on all edges of the square at a rate guaranteeing no aliasing. Shown is the data from the bottom edge of the square. We can see that under sampling in $t$ has resulted in the Fourier Transform of $M f$ being folded into the band limit region. Under sampling in $t$ shifts large frequencies from $\mathcal{F}(M f)$, thus producing the blurred image we see in the right of Figure 9.

Suppose we only average the time data in $M f(t, y)$. This corresponds to taking $b=0$ above to give $p_{0}(x, \xi)=\psi\left(a|\xi|_{g}^{2}\right)$. This symbol takes its minimum values where $|\xi|_{g}^{2}=c^{2}(x) g_{0}^{i j} \xi_{i} \xi_{j}$ is maximized. 


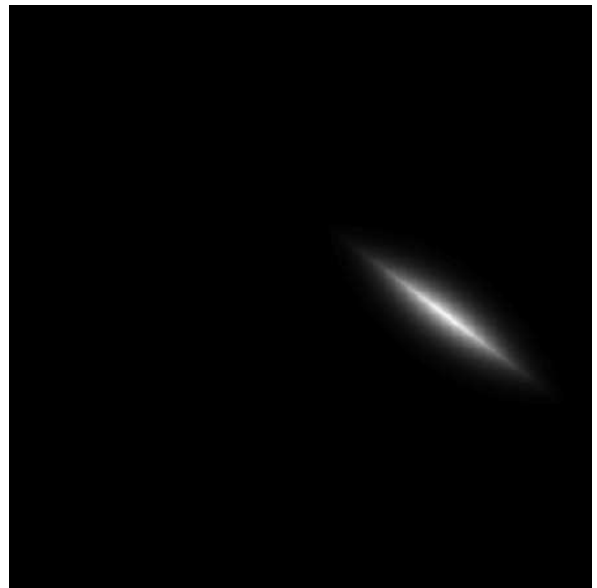

Original Image

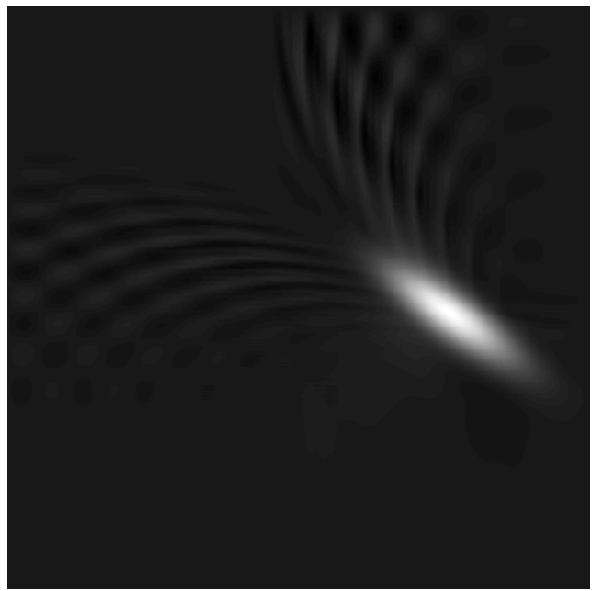

Reconstructed Image

Figure 11. Original and reconstructed image of a smooth approximation of an line segment. Here we have under sampled in $y$. This has resulted in some blurring, but also in high frequency artifacts.

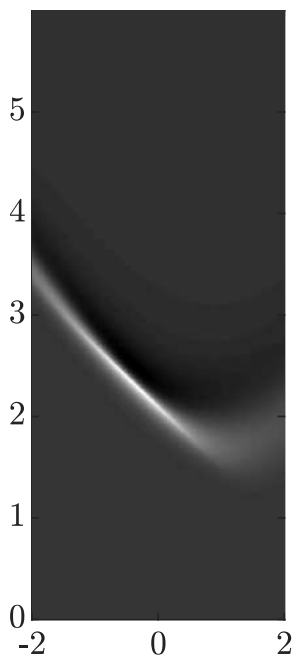

Collected Data

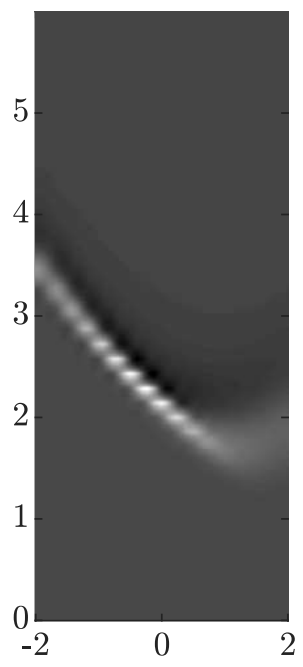

Downsampled Data
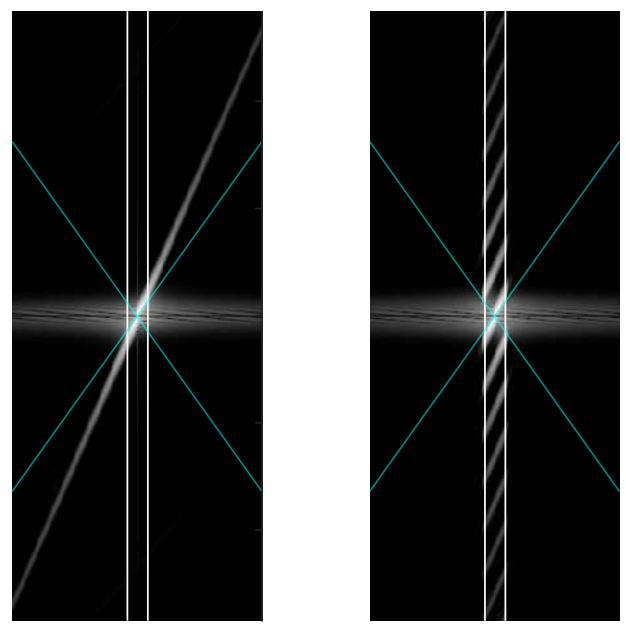

Fourier Transform Fourier Transform of of Collected Data Undersampled Data

Figure 12. Collected data and Fourier transform along with under sampled data in $y$ for example given in Figure 11. In contrast to when we under sample in $t$, we see that high frequencies in $\mathcal{F}(M f)$ are not necessarily eliminated when we under sample in $y$, but there is a phase shift. This results in more high frequency artifacts in the image on the right in Figure 11 .

Assuming for a moment that $g$ is Euclidean, this means that we expect more blurring at points $(x, \xi)$ where the wave speed is "fast". Additionally, we expect $\operatorname{singularities}(x, \xi)$ with large frequencies $|\xi|$ to be blurred more than smaller frequencies where the wave speed is the same. These effects can both be seen in Figure 13 . 


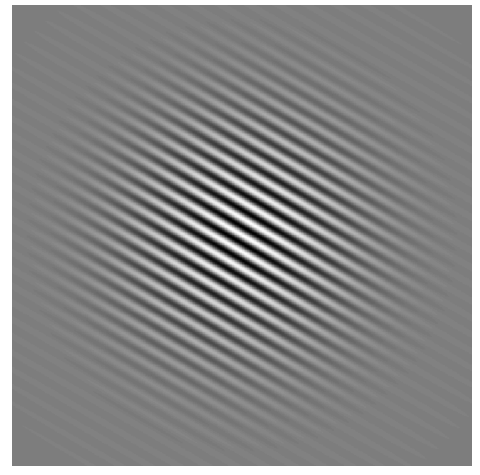

Original Image

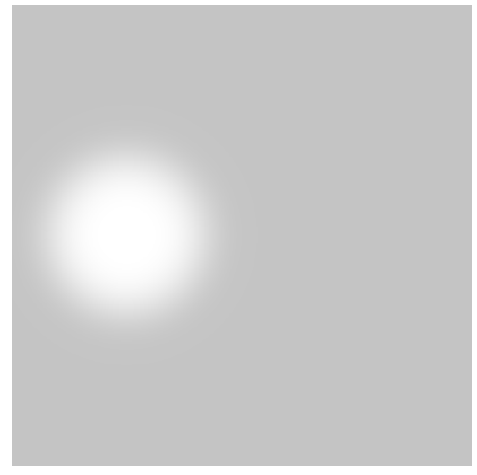

Wave speed with fast spot

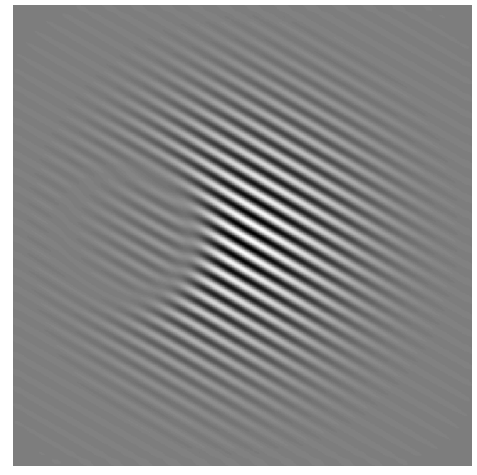

Reconstructed image with data averaged in $t$ variable.

Figure 13. Reconstructed image from data that has been averaged in time variable. We can see that the reconstructed image is most blurred at the points where the speed $c(x)$ is fast, and there is less blurring where $c(x)=1$.

Suppose now that we only average data in the spatial variable $y$. This corresponds to taking $a=0$ above and we get the principle symbol of $p_{0}$ to be

$$
p_{0}(x, \xi)=\frac{1}{2}\left(\psi\left(b\left|\dot{\gamma}_{x, \xi}^{\prime}\left(s_{+}(x, \xi)\right)\right|^{2}\right)+\psi\left(b\left|\dot{\gamma}_{x, \xi}^{\prime}\left(s_{-}(x, \xi)\right)\right|^{2}\right)\right) .
$$

Here the norm is the induced norm on the boundary, which we have noted in this paper as $g_{0, \partial \Omega}$. This symbol takes its smallest values when $\left|\dot{\gamma}_{x, \xi}^{\prime}\left(s_{ \pm}(x, \xi)\right)\right|^{2}$ is large, i.e. when the geodesic issued from $(x, \xi)$ intersects the boundary $\partial \Omega$ at a large angle. In addition, we expect singularities that hit the boundary $\partial \Omega$ perpendicularly to be affected far less by averaging of data in the $y$ variable. In addition, because $\left|\dot{\gamma}_{x, \xi}^{\prime}\left(s_{ \pm}(x, \xi)\right)\right|^{2}=|\xi|_{g}^{2} \cos ^{2}\left(\theta_{ \pm}\right)$where $\theta_{ \pm}$is the angle between $\dot{\gamma}_{x, \xi}^{\prime}\left(s_{ \pm}(x, \xi)\right)$ and $\dot{\gamma}_{x, \xi}\left(s_{ \pm}(x, \xi)\right)$ we expect to see more blurring at points with faster speeds or higher frequency. For constant speeds $c$, the effect of averaging data in $t$ is uniform in $\Omega$, but the effect is local for averaging in $y$, due to the blurring depending on the angle of intersection made by geodesics. In addition, with a variable speed singularities in "slow spots" of $c$ will have higher resolution when blurring $M f(t, y)$ in the $y$-data, but their resolution will still depend on how geodesics hit the boundary. The result is a roughly uniform blurring in fast spots of $c$, and local blurring elsewhere in the image depending on the geometry determined by $c^{-2} g_{0}$. This can be seen in Figure 14 below.

\section{Anti-Aliasing}

We can use the above discussion to propose an anti-aliasing scheme. Averaging the measured data $M f(t, y)$ in the space variable can be accomplished in practice in many ways, whether by using small averaging detectors, or by vibrating the boundary $\partial \Omega$ where we are taking pointwise measurements. We know then that this can be modeled by applying the $h$ - $\Psi$ DO $Q_{h}$ to $M f$ where is as in the previous section. This then allows us to say that $Q_{h} M f(t, y)=M P_{h} f(t, y)+O\left(h^{\infty}\right) f$. In other words, by averaging the data in $y$, we measure $P_{h} f(x, \xi)$, where $P_{h}$ is an $h$ - $\Psi$ DO with principle symbol $p_{0}(x, \xi)=q_{0} \circ C(x, \xi)$ and $C$ is the canonical relation of $M$, plus some error term with low order frequencies. We then expect that if we average $M f(t, y)$ in the $y$ variable before sampling, this should remove some of the shifting aliasing artifacts that appear when $M f(t, y)$ has 


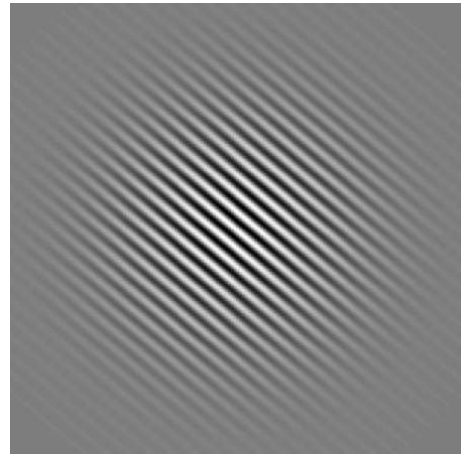

Original Image

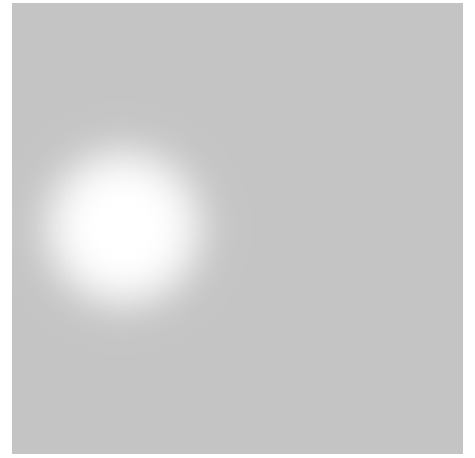

Wave Speed with fast spot

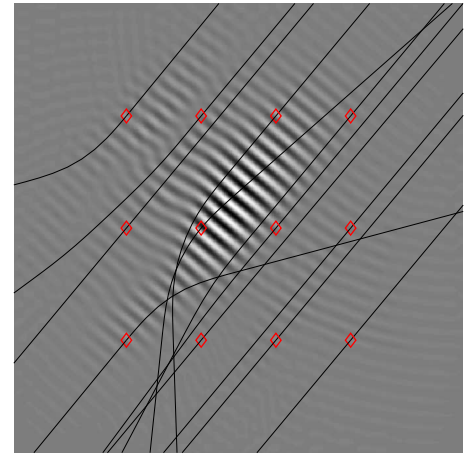

Reconstructed image with data averaged in $y$ variable.

Figure 14. Reconstructed image from data that has been averaged in space variable. We can see from the drawn in geodesics, that singularities that hit the boundary at a larger angle to the normal vector to the boundary are blurred more in the reconstructed image after averaging the collected data. Meanwhile, those singularities that hit the boundary nearly perpendicularly are largely unaffected by the averaging of the data, at least on one side.

been under sampled in $y$, perhaps at the cost of some loss of resolution. See Figure 15 for an example of this anti-aliasing scheme in action.

As a final note, we point out that under sampling in the time variable $t$ can cause data in $\mathcal{F}(M f)$ to shift outside of the characteristic cone, and from this, one should be able to recover some high frequency singularities from data $M f(t, y)$ under sampled in $t$ by shifting these singularities back out into the characteristic cone where they necessarily originated (see Figure 4). However, we can only recover a small fraction of the high frequency singularities in this way uniquely in special cases, and in general we cannot recover the singularities without adding high frequency artifacts to the reconstructed image.

\section{REFERENCES}

[1] S. Bartels. Numerical Approximation of Partial Differential Equations. Vol. 64. Texts in Applied Mathematics. Switzerland: Springer International Publishing, 2016. ISBN: 9783319323541.

[2] P. Burgholzer et al. "Thermoacoustic tomography using a fiber-based Fabry-Perot interferometer as an integrating line detector". In: Photons Plus Ultrasound: Imaging and Sensing 2006: The Seventh Conference on Biomedical Thermoacoustics, Optoacoustics, and Acoustooptics. Ed. by A. A. Oraevsky and L. V. Wang. Vol. 6086. International Society for Optics and Photonics. SPIE, 2006, pp. $434-442$. DOI: $10.1117 / 12.644047$, URL: https : //doi.org/10.1117/12.644047.

[3] R. Courant, K. Friedrichs, and H. Lewy. On the partial difference equations of mathematical physics. Tech. rep. 1959. uRL: http://www.dtic.mil/docs/citations/AD0236290.

[4] H. Grün et al. "Photoacoustic tomography using a fiber based Fabry-Perot interferometer as an integrating line detector and image reconstruction by model-based time reversal method". In: Novel Optical Instrumentation for Biomedical Applications III. Optical Society of America, 2007, pp. 6631-6636. DOI: 10.1364/ECB0.2007.6631_6. URL: http://www.osapublishing. org/abstract.cfm?URI=ECBO-2007-6631_6. 


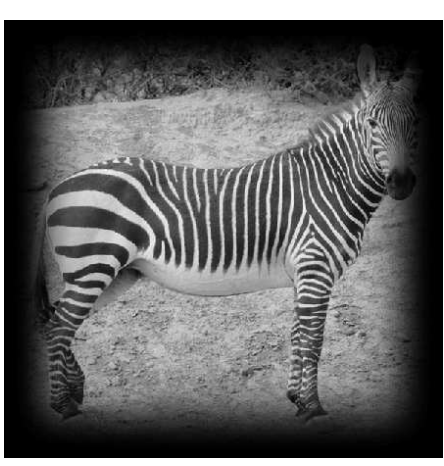

Original Image

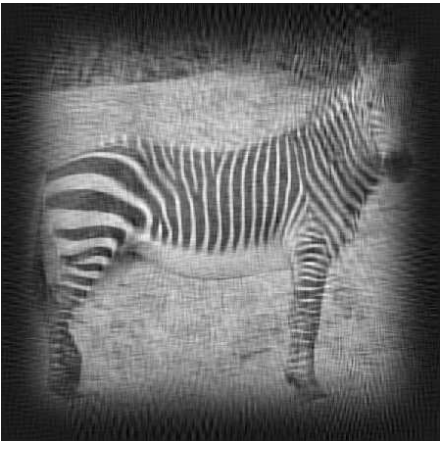

Reconstruction from undersampled $y$ data

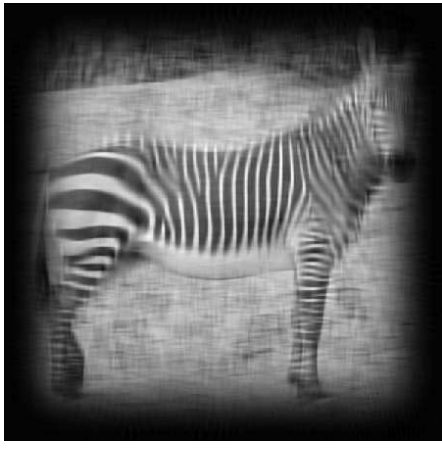

Reconstruction using anti-aliasing scheme

Figure 15. Example showing anti-aliasing scheme in which we first average the data $M f(t, y)$ in the $y$ variable and then sample this blurred version given by $Q_{h} M f(t, y)$ in the above notation. We can see that some of the aliasing artifacts have been removed at the cost of some loss of resolution.

[5] M. Haltmeier. "Thermoacoustic computed tomography with large planar receivers". In: Inverse Problems 20.5 (2004), pp. 1663-1673. ISSN: 0266-5611.

[6] M. Haltmeier et al. "Thermoacoustic tomography and the cicular radon transform: exact inversion formula". In: Mathematical Models and Methods in Applied Sciences 17.4 (2007), pp. 635-655. ISSN: 0218-2025.

[7] Y. Hristova. "Time reversal in thermoacoustic tomography - an error estimate". In: Inverse Problems 25.5 (2009), pp. 055008, 14. ISSN: 0266-5611. DOI: 10.1088/0266-5611/25/5/ 055008. URL: https://doi-org.ezproxy.lib.purdue.edu/10.1088/0266-5611/25/5/ 055008 .

[8] Y. Hristova, P. Kuchment, and L. Nguyen. "Reconstruction and time reversal in thermoacoustic tomography in acoustically homogeneous and inhomogeneous media". In: Inverse Problems 24.5 (2008), pp. 055006, 25. ISSN: 0266-5611. DOI: 10.1088/0266-5611/24/5/055006. URL: https://doi-org.ezproxy.lib.purdue.edu/10.1088/0266-5611/24/5/055006.

[9] R. A. Kruger et al. "Thermoacoustic CT". In: 2000 IEEE MTT-S International Microwave Symposium Digest (Cat. No.00CH37017). Vol. 2. 2000, pp. 933-936. DOI: 10.1109/MWSYM. 2000.863510 .

[10] R. A. Kruger, D. R. Reinecke, and G. A. Kruger. "Thermoacoustic computed tomographytechnical considerations". In: Medical Physics 26.9 (1999), pp. 1832-1837. ISSN: 00942405. DOI: $10.1118 / 1.598688$.

[11] P. Kuchment. The Radon Transform and Medical Imaging. Vol. 85. CBMS-NSF regional conference series in applied mathematics. 2014. ISBN: 1611973287.

[12] L. A. Kunyansky. "Thermoacoustic tomography with detectors on an open curve: an efficient reconstruction algorithm". In: Inverse Problems 24.5 (2008), p. 55021. ISSN: 0266-5611.

[13] R. J. Marks. Introduction to Shannon Sampling and Interpolation Theory. Springer Texts in Electrical Engineering. 1991. ISBN: 1461397103.

[14] A. A. Oraevsky et al. "Laser-based optoacoustic imaging in biological tissues". In: LaserTissue Interaction V; and Ultraviolet Radiation Hazards. Vol. 2134. International Society for Optics and Photonics. 1994, pp. 122-129. 
[15] D. P. Petersen and D. Middleton. "Sampling and reconstruction of wave-number-limited functions in N-dimensional Euclidean spaces". In: Information and Control 5 (1962), pp. 279-323. ISSN: 0019-9958.

[16] P. Stefanov. Semiclassical Sampling and Discretization of Certain Linear Inverse Problems. 2018.

[17] P. Stefanov and G. Uhlmann. "Thermoacoustic tomography with variable sound speed". In: Inverse Problems 25.7 (2009). ISSN: 0266-5611.

[18] P. Stefanov and Y. Yang. "Thermo- and photoacoustic tomography with variable speed and planar detectors". In: SIAM Journal on Mathematical Analysis 49.1 (2017), pp. 297-310. ISSN: 0036-1410.

[19] G. Strang. The Wave Equation and Staggered Leapfrog. 2006. URL: http://math.mit.edu/ classes/18.086/2006/am53.pdf.

[20] M. E. Taylor. Geometrical Optics and Fourier Integral Operators. Princeton University Press, 1981, pp. 146-191.

[21] K. Wang and M. A. Anastasio. "Photoacoustic and thermoacoustic tomography: image formation principles". In: Handbook of mathematical methods in imaging. Vol. 1, 2, 3. Springer, New York, 2015, pp. 1081-1116.

[22] G. Zangerl, O. Scherzer, and M. Haltmeier. "Circular integrating detectors in photo and thermoacoustic tomography". In: Inverse Problems in Science and Engineering 17.1 (2009), pp. 133-142. ISSN: 1741-5977.

[23] M. Zworski. Semiclassical Analysis. Vol. 138. Graduate studies in mathematics. Providence, R.I.: American Mathematical Society, 2012. ISBN: 0821883208. 


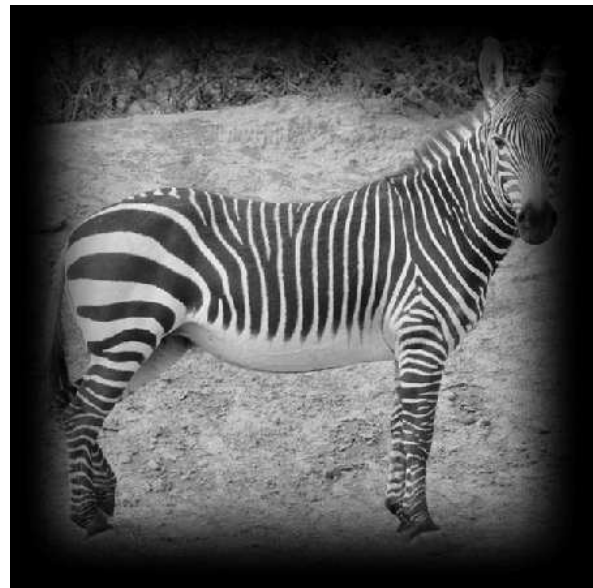

Original Image

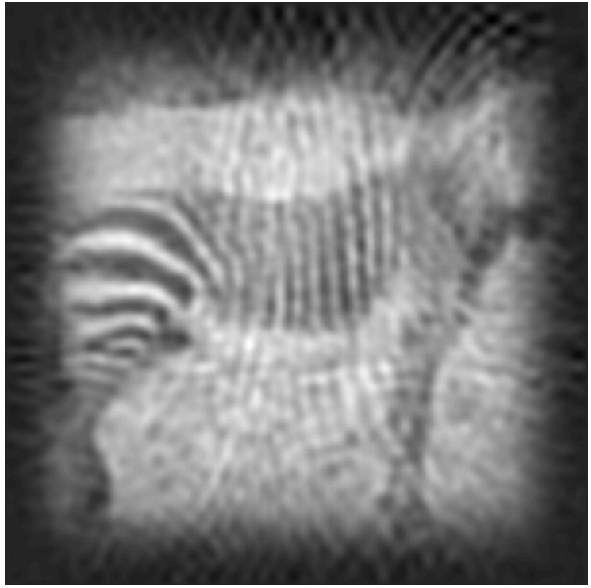

Reconstructed Image

(Undersampled in $t$ )

Figure 16. Image of a zebra along with reconstruction from under sampled (in $t$ ) data. The wave speed here is constant. High frequencies are lost due to this under sampling and the result is a heavily blurred image with aliasing artifacts.

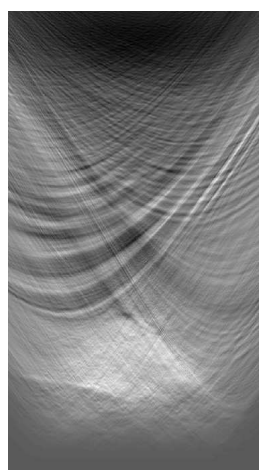

Collected Data (Top edge)

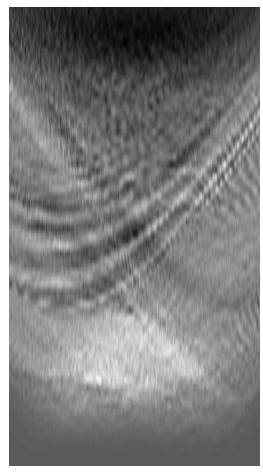

Undersampled Data

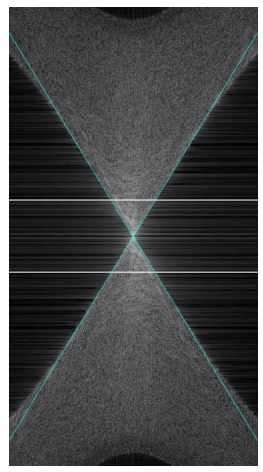

$\mathcal{F}(M f)$

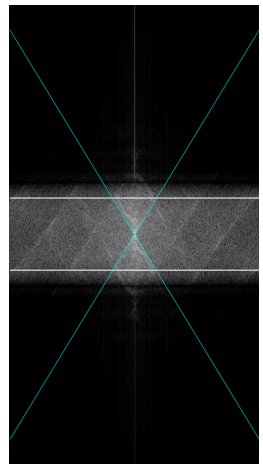

$\mathcal{F}(M f)$

(undersampled)

Figure 17. Collected data and under sampled data in $t$ along with the associated Fourier transform data for the zebra image above. Note that the high frequencies in $\mathcal{F}(M f)$ have be shifted so that they are approximately in the band $-\pi / s_{t}<\tau<\pi / s_{t}$, which is what results in the blurring in the reconstruction. 


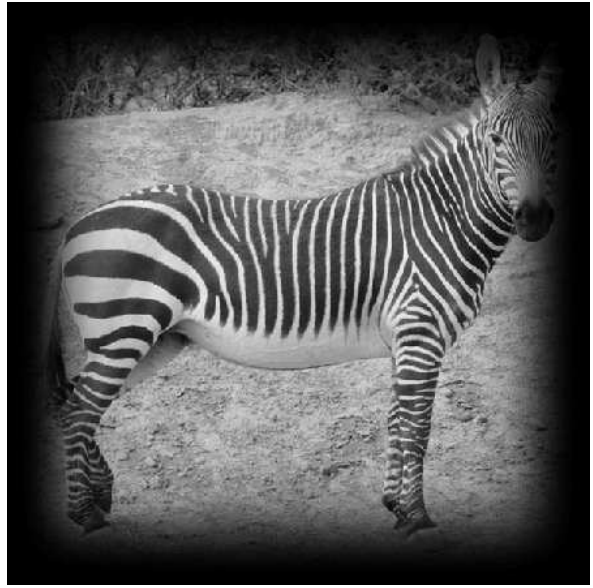

Original Image

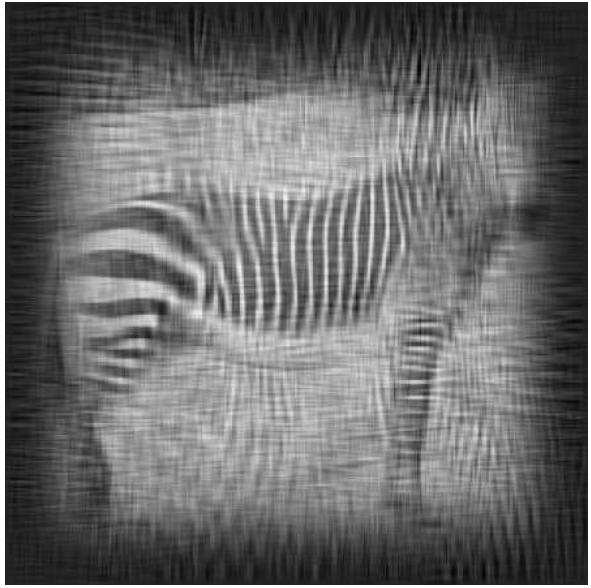

Reconstructed Image

(Undersampled in $y$ )

Figure 18. Image of a zebra along with reconstruction from under sampled (in $y$ ) data. The wave speed here is constant. Note that the singularities that hit the boundary of the square nearly perpendicularly are preserved, but there are also a lot of high frequency artifacts in the reconstructed image.

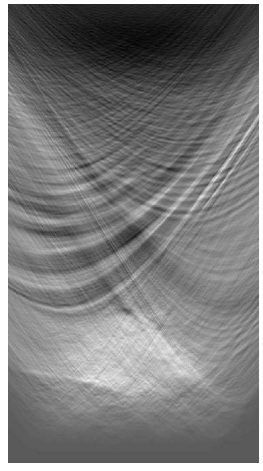

Collected Data (Top edge)

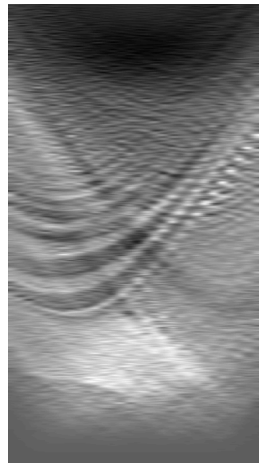

Undersampled

Data

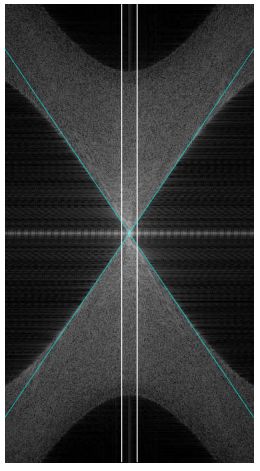

$\mathcal{F}(M f)$

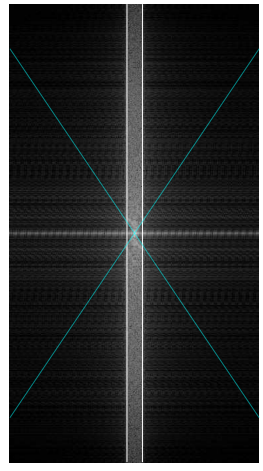

$\mathcal{F}(M f)$

(undersampled)

Figure 19. Collected data and under sampled data in $y$ along with the associated Fourier transform data for the zebra image above. Under sampling has resulted in the shifting of frequencies in $\mathcal{F}(M f)$ so that $-\pi / s_{y^{j}}<\eta<\pi / s_{y^{j}}$. This moves high frequencies but does not destroy them, which is what causes the high frequency artifacts in the reconstructed image above. 